\title{
Quiste hepático gigante secundario a trauma abdominal severo. Hallazgo a 10 años y revisión de la literatura
}

\author{
Giant liver cyst consequent to severe abdominal trauma. \\ Finding after 10 years. Literature review \\ Pablo Magaña-Mainero, ${ }^{*}$ Daniel Garay-Lechuga, ${ }^{*}$ \\ Rafael Jiménez-Martínez, ${ }^{\ddagger}$ Juan Carlos Vázquez-Minero ${ }^{\S}$
}

\begin{abstract}
Palabras clave:
Hígado hepático, postraumático, quiste gigante.

Keywords: Liver, post-traumatic, giant cyst.
\end{abstract}

* Cirugía General. American British Cowdray Medical Center, I.A.P. Ciudad de México, México. ¥ Cirugía General. Cruz Roja Mexicana, I.A.P. Ciudad de México, México.

$\$$ Cirugía Cardiotorácica. Cruz Roja Mexicana, I.A.P. Ciudad de México, México.

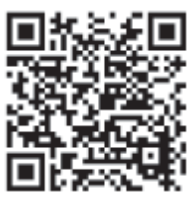

\section{RESUMEN}

Introducción: El trauma abdominal es uno de los motivos más frecuentes de ingreso al servicio de urgencias. El trauma hepático representa la principal causa de muerte en el trauma abdominal cerrado con una tasa de hasta $15 \%$. Los quistes hepáticos son la secuela menos frecuente. Objetivo: Presentar un caso clínico raro y revisión de la literatura. Masculino de 40 años de edad, con antecedente de accidente automovilístico que requirió cirugía en la cual se realizó resección hepática. 10 años después, acude al servicio de urgencias policontundido por agresión de terceras personas, durante el abordaje radiológico se detecta un quiste hepático gigante. Material y métodos: Se hizo una revisión de la literatura internacional a través de buscadores electrónicos, obteniendo sólo cinco artículos originales limitados a reportes de caso. Resultado: No existe un algoritmo fidedigno a seguir para el manejo de los quistes hepáticos postraumáticos debido a que la evidencia descrita se limita a reportes de casos con abordajes no estandarizados y particulares de cada caso. Conclusiones: Los quistes hepáticos postraumáticos representan un porcentaje bajo en la incidencia de la patología hepática y el tratamiento invasivo se reserva a aquellos pacientes que muestran complicaciones locales. En nuestro caso, el paciente carecía de sintomatología o complicaciones ocasionadas por el quiste a pesar del tamaño y tiempo de evolución.

\section{ABSTRACT}

Introduction: Abdominal trauma represents one of the most frequent reasons for admission to the emergency department. Hepatic trauma represents the main cause of death in closed abdominal trauma with rates as high as $15 \%$. Hepatic cysts are the less frequent sequel. Objective: To present a rare case and a review of the literature. 40-year-old male with a history of a car accident that required surgery in which hepatic resection was performed. 10 years later, he is admitted in the emergency department with multiple contusions after the attack by third persons, during the radiologic approach a giant liver cyst is found. Material and methods: A review of the international literature was made through electronic search engines, obtaining only five original articles limited to case reports. Result: There's no algorithm to follow in the management of post-traumatic liver cyst given that the reported evidence is resumed to case reports without standardized approaches and particular managements of each case. Conclusions: Post-traumatic hepatic cysts represent a low percentage in the incidence of liver disease and invasive treatment is reserved for those patients who present local complications. In our case, the patient lacked symptoms or complications caused by the cyst despite the size and time of evolution.

\section{INTRODUCCIÓN}

$\mathrm{E}$ I trauma hepático es frecuente tanto en el trauma penetrante como en el trauma contuso, la mortalidad en el trauma hepático depende del grado de lesión del mismo, siendo las lesiones grado VI frecuentemente mortales. ${ }^{1}$ El trauma hepático suele tener secuelas, entre

Citar como: Magaña-Mainero P, Garay-Lechuga D, Jiménez-Martínez R, Vázquez-Minero JC. Quiste hepático gigante secundario a trauma abdominal severo. Hallazgo a 10 años y revisión de la literatura. Cir Gen. 2020; 42(1): 19-23. doi: $10.35366 / 92707$ 
las cuales se encuentran: fuga biliar, abscesos, necrosis isquémica, etc. Los quistes hepáticos son la secuela menos frecuente.

\section{PRESENTACIÓN DEL CASO}

Hombre de 40 años de edad, con antecedentes de accidente automovilístico 10 años previos a su ingreso, en el que requirió intervención quirúrgica por traumatismo abdominal cerrado, con colecistectomía y resección hepática, se desconoce la magnitud y resección intestinal y lesión vascular femoral. En esta ocasión es llevado por paramédicos al servicio de urgencias por mostrar agresión de terceras personas, presentaba contusiones múltiples en cabeza, tórax, abdomen y miembros torácicos, posterior a lo cual es arrollado por automóvil con paso del vehículo sobre el abdomen.

A su ingreso se mostraba ansioso, alerta, orientado; valoración inicial ATLS (Advanced Trauma Life Support), sin compromiso hemodinámico, neurológicamente sin déficit, tórax con dermoabrasiones, campos ventilados de manera bilateral, hipoventilación basal de hemitórax derecho, abdomen con marcas cutáneas de neumáticos, blando, depresible con dolor a la palpación generalizada profunda, datos de irritación peritoneal en cuadrantes

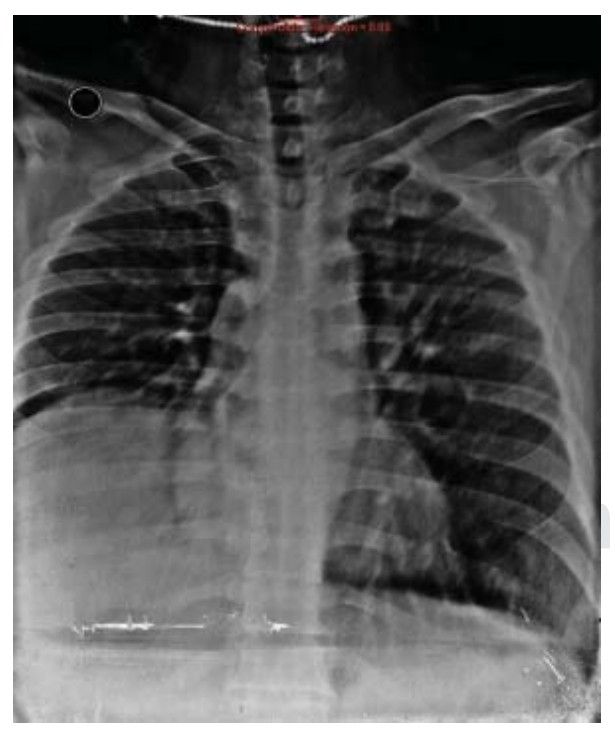

Figura 1: Radiografía AP de tórax con elevación del diafragma derecho por causa a determinar. inferiores, extremidades con dermoabrasiones en antebrazos. Se realizan placas de protocolo de ATLS, se observó en la radiografía de tórax (Figura 1) hemitórax derecho con elevación del hemidiafragma, sin neumotórax o derrame pleural, se efectúa FAST (Focused Abdominal Sonography for Trauma) primario sin revelar líquido en pericardio, espacio hepatorrenal, esplenorrenal o en pelvis, se realiza tomografía axial computarizada simple y contrastada toraco-abdomino-pélvica (Figuras 2 y 3) en la que se observa imagen compatible con quiste hepático simple de 12.6 x $14.7 \mathrm{~cm}$.

A causa del hallazgo tomográfico el paciente permaneció en vigilancia, a las 48 horas de su estancia intrahospitalaria se efectuaron controles de laboratorio y de imagen (Figura 4), sin cambios significativos con respecto a su ingreso. No se evidenció conversión hemorrágica de quiste hepático. A su egreso, se observaba paciente con mejoría sintomatológica, sin complicaciones.

\section{REVISIÓN}

La enfermedad quística del hígado tiene origen diverso y el diferencial incluye patologías como abscesos bacterianos y parasitarios, bilomas, cistadenomas y cistadenocarcinomas. También se pueden clasificar de acuerdo con su origen como congénito o adquirido. Dentro de los adquiridos la enfermedad traumática y neoplásica representa el menor porcentaje, dejando a los quistes postraumáticos como una entidad descrita de menos de $0.5 \% .^{2}$ El diagnóstico se logra principalmente mediante hallazgos transoperatorios debido a complicaciones de los mismos y el resto de forma incidental durante abordajes imagenológicos. ${ }^{3}$ El tratamiento tiene la tendencia al manejo conservador; sin embargo, existe controversia en cuanto al mejor tratamiento y la disponibilidad del mismo. ${ }^{4}$

Se realizó una búsqueda de la literatura en PubMed de artículos originales en español e inglés con las palabras MESH en forma entrecruzada "Liver"[Mesh], "Cysts"[Mesh], "Post-traumatic"[Word] con objeto de revisar la literatura en cuanto a incidencia, diagnóstico, etiopatogenia y tratamiento. Se obtuvieron cinco artículos originales publicados entre los años 1996 y 2015, los cuales corresponden a 


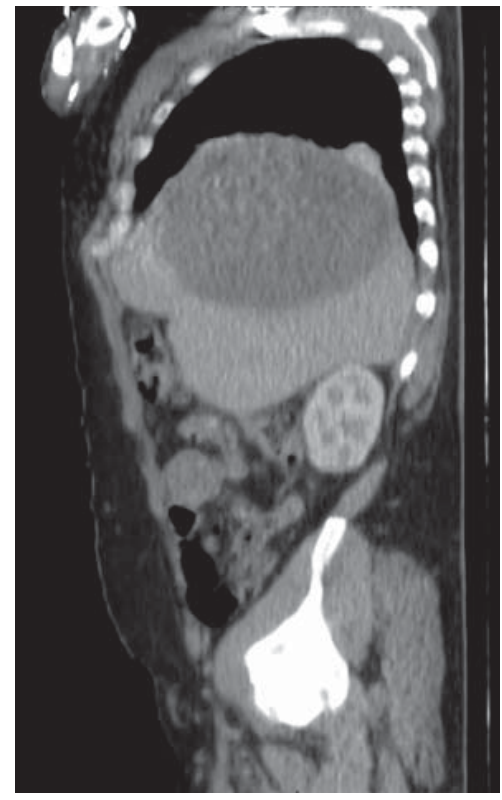

Figura 2: Tomografía computarizada de tórax y abdomen corte sagital paramedial con lesión quística hepática con desplazamiento de segmentos VII y VIII y diafragma a región intratorácica.

reportes de casos. Se elaboró una tabla comparativa entre los mismos (Tabla 1).

El hígado representa el órgano principalmente involucrado en el trauma abdominal cerrado. ${ }^{5}$ Los quistes hepáticos no infecciosos son una entidad descrita en un inicio en 1937 por Sanders, ${ }^{6}$ de los cuales la presentación más común es de origen congénito, de este modo, los quistes adquiridos secundarios a una lesión traumática representan la variedad menos frecuente. ${ }^{7}$ Se cuenta con una cantidad escasa de casos descritos en la literatura internacional, principalmente de países asiáticos y en pacientes pediátricos. La localización más frecuente es en el lóbulo derecho y son lesiones uniloculares. El hallazgo incidental es la presentación más frecuente con el antecedente de trauma, esto debido al manejo no quirúrgico cada vez más frecuente en pacientes con lesiones hepáticas de grado IV y V. ${ }^{4,6}$ Sin embargo, otras series no han encontrado correlación en el grado del trauma y la formación de quistes. ${ }^{7}$ En nuestro caso no se contaba con registros del grado de lesión, ni de la tasa de crecimiento del quiste, ya que el paciente durante los 10 años previos a su admisión se mantuvo totalmente asintomático, y sólo fue diagnosticado como hallazgo dentro del protocolo de estudio del trauma abdominal.

La formación de estos quistes es secundaria a lesión traumática, ocasiona fuga de bilis y sangre que resulta en un pseudoquiste (no tiene epitelio), el sangrado suele autolimitarse a través de la coagulación, mientras que el flujo de bilis continúa y por lo tanto, propicia el crecimiento del quiste. ${ }^{8}$ Por esta razón, los síntomas suelen ser tardíos. ${ }^{9}$ En nuestro caso, a pesar del tamaño y el tiempo de evolución, el paciente negó sintomatología alguna, que de no ser por este incidente actual no se hubiera diagnosticado.

La presentación clínica es variada, ya que la mayoría resuelven de manera espontánea y otro porcentaje progresan asintomáticos hasta provocar síntomas compresivos, los cuales requieren tratamiento. ${ }^{4} \mathrm{~A}$ pesar de esto, com-
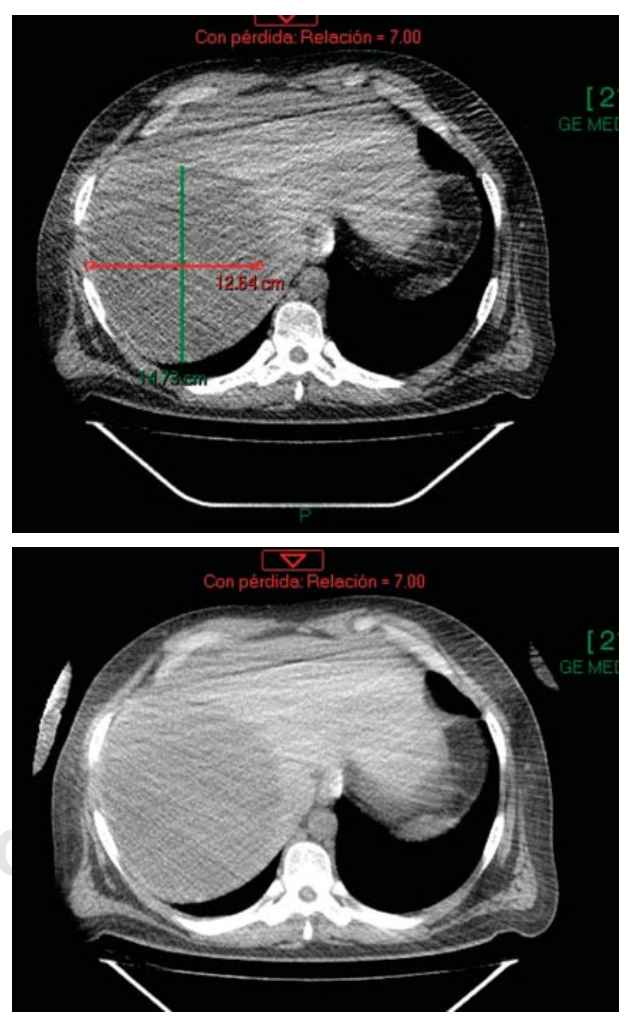

Figura 3: Tomografía computarizada simple de tórax y abdomen, corte axial con quiste hepático de $12.64 x$ $14.73 \mathrm{~cm}$ lóbulo derecho. 

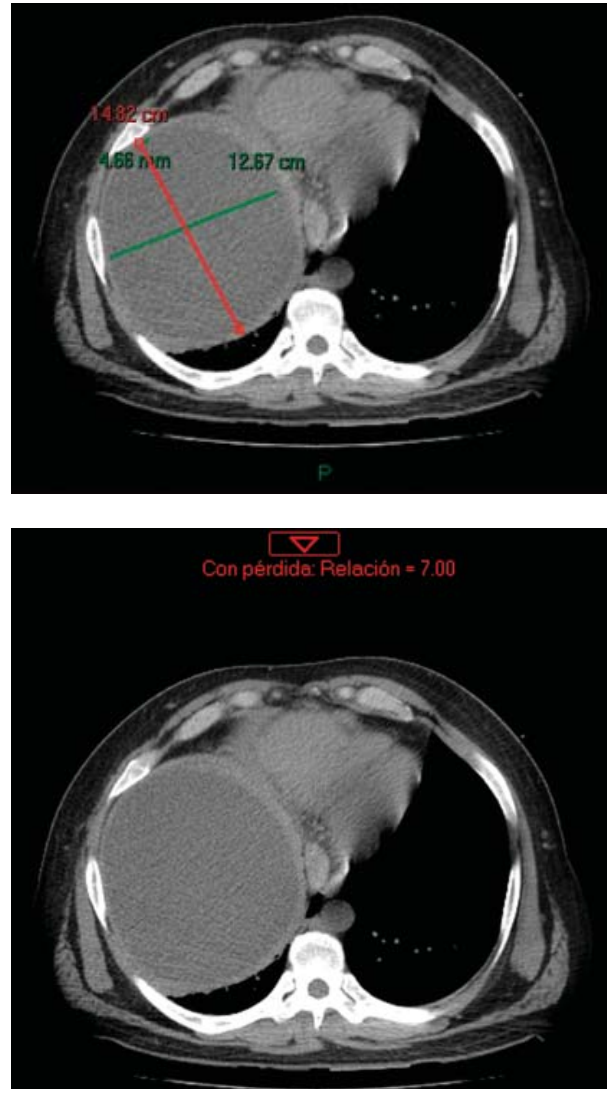

Figura 4: Tomografía computarizada simple de tórax y abdomen, corte axial de control a las 48 horas sin aumento de volumen. plicaciones como ictericia obstructiva, choque hemorrágico y peritonitis biliar suelen ser poco frecuentes como en este caso.

Se ha descrito la regresión espontánea de los quistes postraumáticos; ${ }^{6}$ sin embargo, en el caso de los sintomáticos, dentro de las opciones terapéuticas se encuentra el drenaje con una recurrencia alta a dos años; asimismo, se ha reportado que una vez drenado el contenido de la fístula, suelen resolver de manera espontánea. ${ }^{4}$ En el caso de los quistes simples, la escisión y destechamiento son opciones terapéuticas viables con una recurrencia de $0-20 \%$ y una mortalidad de hasta $5 \%$. En el caso de nuestro paciente se optó por el tratamiento conservador dada la ausencia de sintomatología y evolución favorable sin conversión hemorrágica.

El quiste hepático postraumático está vinculado a la lesión de conducto biliar, una proporción se ve ligada a colecistectomía, pero la etiología de la mayoría está ligada a un antecedente de trauma.

\section{CONCLUSIONES}

La opción terapéutica menos mórbida es el drenaje percutáneo, indicado en pacientes con sintomatología principalmente compresiva a nivel abdominal. No existe hoy

Tabla 1: Revisión de la literatura correspondiente a quiste hepático postraumático.

\begin{tabular}{|c|c|c|c|c|c|c|}
\hline Estudio & $\begin{array}{l}\text { Paciente } \\
\text { (edad) }\end{array}$ & $\begin{array}{l}\text { Tiempo de } \\
\text { evolución }\end{array}$ & $\begin{array}{c}\text { Estado } \\
\text { hemodinámico }\end{array}$ & $\begin{array}{l}\text { Tamaño } \\
\text { (cm) }\end{array}$ & $\begin{array}{c}\text { Complicaciones } \\
\text { locales }\end{array}$ & Manejo \\
\hline Chen et al. ${ }^{2}$ & $\begin{array}{l}\text { Femenino. } \\
63 \text { años }\end{array}$ & 1 año & Estable & $12 \times 10$ & $\begin{array}{c}\text { Compresión extrínseca } \\
\text { de estómago }\end{array}$ & Destechamiento \\
\hline Chuang et $\mathrm{al}^{6}$ & $\begin{array}{l}\text { Femenino. } \\
7 \text { años }\end{array}$ & $\begin{array}{c}5 \text { años } \\
8 \text { meses }\end{array}$ & Estable & $11 \times 11$ & $\begin{array}{l}\text { Dolor } \\
\text { crónico* }\end{array}$ & Resección \\
\hline Dalal et al. ${ }^{10}$ & $\begin{array}{l}\text { Masculino. } \\
22 \text { años }\end{array}$ & 3 meses & Estable & $12 \times 10$ & $\begin{array}{c}\text { Dolor } \\
\text { crónico* }\end{array}$ & $\begin{array}{c}\text { Drenaje } \\
\text { percutáneo }\end{array}$ \\
\hline Singh et al. ${ }^{9}$ & $\begin{array}{l}\text { Femenino. } \\
34 \text { años }\end{array}$ & 2 meses & Estable & $10 \times 10$ & $\begin{array}{c}\text { Compresión extrínseca } \\
\text { de estómago }\end{array}$ & Destechamiento \\
\hline Sharma et al. ${ }^{5}$ & $\begin{array}{c}\text { Femenino. } \\
18 \text { meses }\end{array}$ & 2 meses & Estable & $10 \times 11$ & $\begin{array}{l}\text { Dolor } \\
\text { crónico* }\end{array}$ & Drenaje abierto \\
\hline
\end{tabular}

* Dolor crónico CIE-11. ${ }^{11}$ 
en día dentro de las clasificaciones actuales un algoritmo definido para el diagnóstico y tratamiento de estas lesiones; sin embargo, la tomografía representa, como en nuestro caso, la herramienta diagnóstica más eficiente y acertada.

El tratamiento resulta controversial y sin indicaciones bien definidas, se hace hincapié en el tratamiento quirúrgico en todo paciente con sintomatología compresiva, dolorosa o gastrointestinal.

\section{REFERENCIAS}

1. Badger SA, Barclay R, Campbell P, Mole DJ, Diamond T. Management of liver trauma. World J Surg. 2009; 33: 2522-2537.

2. Chen BK, Gamagami RA, Kang J, Easter D, Lopez T. Symptomatic post-traumatic cyst of the liver: treatment by laparoscopic surgery. J Laparoendosc Adv Surg Tech A. $2001 ; 11: 41-42$.

3. Asuquo M, Nwagbara V, Agbor C, Otobo F, Omotoso A Giant simple hepatic cyst: a case report and review of relevant literature. Afr Health Sci. 2015; 15: 293-298.

4. Zinner M, Ashley JS. Maingot's abdominal operations. 12th edition. New York: McGraw Hill Professional; 2012.

5. Sharma DD, Shukla AK, Chaturvedi V, Jangid M, Raipuria G. Post traumatic pseudocyst of liver. JCR. 2015; 5: 271-273.

6. Chuang JH, Huang SC. Posttraumatic hepatic cyst--an unusual sequela of liver injury in the era of imaging. J Pediatr Surg. 1996; 31: 272-274.

7. Sugimoto T, Yoshioka T, Sawada $Y$, Sugimoto $H$, Maemura K. Post-traumatic cyst of the liver found on CT scan--a new concept. J Trauma. 1982; 22: 797-800.
8. Christopher F. Rupture of the liver. Ann Surg. 1936; 103: 461-463.

9. Singh KK, Nizarudeen A, Sulfikar MS, Maheshwaran A, George D. Post-traumatic (haemorrhagic)liver cyst. Indian J Surg. 2013; 75: 425-427.

10. Dalal S, Garg P, Rohilla P. Post-traumatic hepatic cyst: an unusual sequel of liver injury. Internet Journal of Surgery. 2008; 8: 1-3.

11. Treede RD, Rief W, Barke A, Aziz Q, Bennett MI, Benoliel R, et al. A classification of chronic pain for ICD-11. Pain. 2015; 156: 1003-1007.

\section{Consideraciones y responsabilidad ética:} Privacidad de los datos. De acuerdo a los protocolos establecidos en el centro de trabajo de los autores, éstos declaran que han seguido los protocolos sobre la privacidad de datos de pacientes preservando su anonimato. El consentimiento informado del paciente referido en el artículo se encuentra en poder del autor.

Financiamiento: No se recibió apoyo financiero para la realización de este trabajo.

Conflicto de intereses: Los autores declaran que no existe ningún conflicto de intereses en la realización del trabajo.

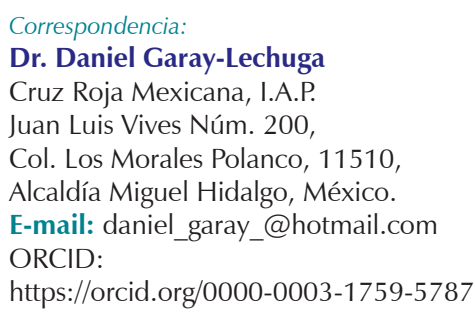

